TRANSACTIONS OF THE

AMERICAN MATHEMATICAL SOCIETY

Volume 361, Number 7, July 2009, Pages 3793-3815

S 0002-9947(09)04875-2

Article electronically published on February 10, 2009

\title{
A PRIORI ESTIMATES OF POSITIVE SOLUTIONS FOR SUBLINEAR ELLIPTIC EQUATIONS
}

\author{
RYUJI KAJIKIYA
}

\begin{abstract}
In this paper, a priori estimates of positive solutions for sublinear elliptic equations are given in terms of thicknesses of domains. To this end, a supersolution is constructed by a composite function of a solution to an ordinary differential equation and a distance function. The results work efficiently in the case where the domain is an exterior or an interior of a convex set.
\end{abstract}

\section{INTRODUCTION}

In this paper, we give a priori estimates of positive solutions for the following problem in terms of a thickness of a domain:

$$
\begin{array}{cc}
-\Delta u=f(u), & u>0, \quad \text { in } \Omega, \\
u=0, & \text { on } \partial \Omega .
\end{array}
$$

Here $\Omega$ is a bounded domain in $\mathbb{R}^{N}$ and $f(s)$ is continuous in $[0, \infty)$ and sublinear, i.e., $f(s) / s$ is strictly decreasing in $(0, \infty)$. It is well-known that if $f(s) / s$ diverges to $\infty$ as $s \rightarrow 0$ and converges to 0 as $s \rightarrow \infty$ or $f(s) \leq 0$ for $s>0$ large, then (1.1), (1.2) has a unique positive solution. This will be seen later in Theorem 1.1. Our problem in this paper is as follows. Estimate the positive solution by an easy and familiar function. By using it, investigate the relation between the size of the positive solution and the thickness of the domain. Our answer is

$$
u(x) \leq v(\operatorname{dist}(x, \partial D), d) \quad \text { in } \Omega,
$$

provided that $\Omega$ lies outside a certain convex set $D$ and lies within a distance $d$ from $\partial D$. The right-hand side in (1.3) forms a composite function of $v(t, d)$ and $\operatorname{dist}(x, \partial \Omega)$, where $v(t, d)$ is a positive solution of the ordinary differential equation,

$$
\left\{\begin{array}{l}
-v^{\prime \prime}=f(v), \quad v(t)>0, \quad \text { in }(-d, d), \\
v(-d)=v(d)=0,
\end{array}\right.
$$

and $\operatorname{dist}(x, \partial D)$ denotes the distance from $x$ to $\partial D$. Another our answer is

$$
u(x) \leq v(\operatorname{dist}(x, \partial D)-d, d) \quad \text { in } \Omega,
$$

Received by the editors August 10, 2007.

2000 Mathematics Subject Classification. Primary 35B45, 35J25; Secondary 35J65.

Key words and phrases. Sublinear elliptic equation, a priori estimates, thickness of domain, positive solution.

This work was supported in part by the Grant-in-Aid for Scientific Research (C) (No. 20540197), Ministry of Education in Japan. 
provided that $\Omega \subset D$ and $D$ is bounded open convex and $d$ is the radius of the maximum ball in $D$. In both cases (1.3) and (1.5), $d$ can be considered as the thickness of $\Omega$. The motivation for our paper is to investigate the positive solution by means of a simple and easy function and to estimate the size of the positive solution by the thickness of the domain. Indeed, using (1.3) and (1.5), we can derive the $L^{\infty}(\Omega)$-estimates of the positive solution $u$,

$$
\begin{gathered}
\|u\|_{\infty} \leq C d^{2 /(1-p)} \quad \text { if } \quad f(u)=u^{p} \text { with } 0<p<1 \\
\|u\|_{\infty} \leq \exp \left(\frac{1}{2}-\frac{\pi^{2}}{4 d^{2}}\right) \quad \text { if } \quad f(u)=-u \log u
\end{gathered}
$$

These inequalities give us the decay estimate of positive solutions as the thickness $d$ tends to zero. Before discussing our results more, we state the known results about the existence and uniqueness of positive solutions. For such a problem, we refer to Brezis-Oswald's result 3 . Their theorem is valid for $f=f(x, u)$ also; however we state it here in case $f=f(u)$ only.

Theorem $1.1\left([3)\right.$. Let $\Omega$ be a bounded smooth domain in $\mathbb{R}^{N}$. Suppose that $f(s) / s$ is strictly decreasing in $(0, \infty)$. Then (i) and (ii) below hold:

(i) (1.1), (1.2) has at most one solution.

(ii) A solution of (1.1), (1.2) exists in $W^{2, p}(\Omega)$ for any $1 \leq p<\infty$ if and only if

$$
a_{\infty}<\lambda_{1}<a_{0}
$$

where $\lambda_{1}$ denotes the first eigenvalue of $-\Delta$ with the zero Dirichlet condition and $a_{0}, a_{\infty}$ are defined by

$$
a_{0}=\lim _{s \rightarrow 0} f(s) / s, \quad a_{\infty}=\lim _{s \rightarrow \infty} f(s) / s .
$$

Our result in this paper is valid for $f(x, u)$ also; however for simplicity of notation and discussions, we consider the non-linear term $f=f(u)$ independent of $x$. See Remark 2.7 for the case $f=f(x, u)$. Theorem 1.1 with $f(x, u)$ has been extended by Taira-Umezu [8] and Bao 2 to a more general form of second order elliptic equations. Amann [1] and de Figueiredo [4] have proved that the comparison theorem works well for the sublinear elliptic equations. They have used it to obtain the existence and the uniqueness of positive solutions. Recently, without any regularity conditions on $f(x, u)$ and $\partial \Omega$, we prove in [6] that the necessary and sufficient condition for the comparison theorem to be valid is that $f(x, u)$ is sublinear.

We organize this paper into seven sections. In Section 2, we state the main results, a priori estimates of solutions and give examples of sublinear elliptic equations. In Section 3, we introduce a comparison theorem for weak supersolutions and subsolutions. In Section 4, we compute the estimates given in examples of Section 2. In Sections 5 and 6 , we prove a priori estimates for the exteriors of convex domains and for the interiors, respectively. In Section 6, we will use a distance function from a point to the boundary of a convex domain. Even if the boundary is smooth, the distance function is not differentiable at some points in the interior of the domain. In Section 7, we will prove that the set of singular points for the distance function is compact and has Lebesgue measure zero. 


\section{MAin RESUlts}

In this section, we state the main results and apply them to sublinear elliptic equations. Suppose the conditions below.

Assumption 2.1. (f1) $f(s)$ is continuous in $[0, \infty)$ and $f(s) / s$ is strictly decreasing in $(0, \infty)$.

(f2) For any $R>0$ there is a $C>0$ such that $(f(t)-f(s)) /(t-s) \geq-C$ for $s, t \in[0, R]$ with $s \neq t$.

(f3) $\lim _{s \rightarrow 0+} f(s) / s=\infty$ and $\lim _{s \rightarrow \infty} f(s) / s \leq 0$.

To deal with various domains $\Omega$, we assume (f3). If $\Omega$ is fixed, it is enough to assume (1.6) instead of (f3). Throughout the paper, we always assume that (f1)(f3) hold and $\partial \Omega$ is smooth. Then (1.1), (1.2) has a unique positive solution by Theorem 1.1. We denote the solution of $(1.1),(1.2)$ by $u(x, \Omega)$ and the solution of (1.4) by $v(t, d)$.

Theorem 2.2. Let $\Omega \subset \mathbb{R}^{N-1} \times(-d, d)$. Then we have

$$
\begin{gathered}
u(x, \Omega) \leq v\left(x_{N}, d\right) \quad \text { for } x=\left(x_{1}, \ldots, x_{N}\right) \in \Omega, \\
\|u(\cdot, \Omega)\|_{\infty} \leq\|v(\cdot, d)\|_{\infty}=v(0, d) .
\end{gathered}
$$

Here $\|\cdot\|_{\infty}$ means the $L^{\infty}$-norm.

Theorem 2.2 is optimal because of the next result.

Theorem 2.3. For $d, R>0$, define

$$
\Omega(R, d) \equiv\left\{\left(x^{\prime}, x_{N}\right) \in \mathbb{R}^{N-1} \times \mathbb{R}:\left|x^{\prime}\right|<R,\left|x_{N}\right|<d\right\} .
$$

Then $u(x, \Omega(R, d))$ converges to $v\left(x_{N}, d\right)$ in $C_{l o c}^{1}\left(\mathbb{R}^{N-1} \times(-d, d)\right)$ as $R \rightarrow \infty$. Moreover, if $f(s)$ is locally Hölder continuous in $[0, \infty)$, then it converges in $C_{l o c}^{2}\left(\mathbb{R}^{N-1} \times(-d, d)\right)$.

We can obtain a sharper estimate than Theorem 2.2 when the domain lies out or in a convex set.

Theorem 2.4. Let $D$ be a bounded convex set in $\mathbb{R}^{N}$. For $d>0$, define

$$
D_{d} \equiv\left\{x \in \mathbb{R}^{N} \backslash \bar{D}: \operatorname{dist}(x, \partial D)<d\right\} .
$$

If $\Omega \subset D_{d}$, then $u(x, \Omega)$ satisfies (1.3).

Theorem 2.5. Let $D$ be bounded open and convex. Let d denote the radius of the maximum ball in $D$. If $\Omega \subset D$, then $u(x, \Omega)$ satisfies (1.5).

Since $\|v(\cdot, d)\|_{\infty}$ converges to zero as $d \rightarrow 0$, which will be proved later on, Theorems $2.2-2.5$ assert that a solution in a thin domain is small. We estimate $v(t, d)$ from above and obtain the next corollary.

Corollary 2.6. Suppose that $f(s)$ is bounded from above in $(0, \infty)$. Let $\bar{f}$ be the supremum of $f(s)$ on $0 \leq s<\infty$. Then

$$
\|u(\cdot, \Omega)\|_{\infty} \leq \bar{f} d^{2} / 2 .
$$

Here $d$ is defined by Theorem 2.2, 2.4 or 2.5. 
Remark 2.7. Let us consider the case where $f(x, s)$ depends on $x$. We assume the following conditions:

(i) $f(x, s) / s$ is strictly decreasing in $s \in(0, \infty)$ for each $x \in \bar{\Omega}$.

(ii) For any $R>0$ there is a $C>0$ such that $(f(x, t)-f(x, s)) /(t-s) \geq-C$ for $x \in \bar{\Omega}$ and $s, t \in[0, R]$ with $s \neq t$.

(iii) Put $F(s)=\max _{x \in \bar{\Omega}} f(x, s)$. Assume that $F(s)$ satisfies (f3).

Then $F(s) / s$ is strictly decreasing. Let $v(t, d)$ be a solution of (1.4) with $f(v)$ replaced by $F(v)$. In this case, a solution $u$ of (1.1) with $f(u)=f(x, u)$ becomes a subsolution of (1.1) with $f(u)=F(u)$ because

$$
-\Delta u=f(x, u) \leq F(u) .
$$

Therefore Theorems 2.2, 2.4 and 2.5 and Corollary 2.6 are still valid with the help of Theorem 3.2 later in the paper.

In the following examples, we explain how Theorem 2.4 or 2.5 takes advantage over Theorem 2.2 when $\Omega$ is an interior or an exterior of a convex set.

Example 2.8. Let $\Omega$ be the annulus $R<|x|<R+\varepsilon$. Then $2(R+\varepsilon)$ is the shortest distance between two parallel hyperplanes in which $\Omega$ is put. Therefore Theorem 2.2 with $d=R+\varepsilon$ asserts that

$$
\|u(\cdot, \Omega)\|_{\infty} \leq v(0, R+\varepsilon) .
$$

However Theorem 2.4 gives $\|u(\cdot, \Omega)\|_{\infty} \leq v(0, \varepsilon)$. This is sharper than (2.2) because $v(0, d)$ is increasing in $d$, which will be proved later in the paper.

Example 2.9. Let $N=2$ and $\Omega$ be an interior of an equilateral triangle with sides of length one. Since the height of $\Omega$ is $\sqrt{3} / 2$, Theorem 2.2 with $d=\sqrt{3} / 4$ gives

$$
\|u(\cdot, \Omega)\|_{\infty} \leq v(0, \sqrt{3} / 4) .
$$

Since the radius of the inscribed circle of $\Omega$ is $\sqrt{3} / 6$, Theorem 2.5 guarantees

$$
\|u(\cdot, \Omega)\|_{\infty} \leq v(0, \sqrt{3} / 6) .
$$

Hence Theorem 2.5 gives a sharper estimate than Theorem 2.2.

In sublinear elliptic equations, a positive solution is the biggest of all solutions. Then Theorems 2.2, 2.4 and 2.5 are still valid for all sign changing solutions. Especially, if $f(s)$ is odd, then these theorems give a priori estimates for $|u(x)|$ where $u(x)$ is not only a positive solution but also any sign-changing solution. Hence we have the next theorem.

Theorem 2.10. Let $f(s)$ be odd and $u(x)$ be any solution of (1.1), (1.2). Then Theorems 2.2, 2.4 and 2.5 and Corollary 2.6 remain valid after replacing $u(x, \Omega)$ by $|u(x)|$.

We apply our theorems to some sublinear elliptic equations and give a priori estimates for the $L^{\infty}$-norm of solutions by the thickness of the domain.

Example 2.11. Consider the Emden-Fowler equation,

$$
\left\{\begin{aligned}
-\Delta u & =u^{p}, \quad u>0, \quad \text { in } \Omega, \\
u & =0, \quad \text { on } \partial \Omega .
\end{aligned}\right.
$$

Here $0<p<1$. The unique positive solution $u$ has an a priori estimate,

$$
\|u(\cdot, \Omega)\|_{\infty} \leq C d^{2 /(1-p)} .
$$


Here $d$ is the thickness of $\Omega$ given by Theorem 2.2, 2.4 or 2.5 , and $C$ is defined by

$$
\begin{aligned}
C & \equiv\left(\sqrt{\frac{1+p}{2}} \int_{0}^{1} \frac{d t}{\sqrt{1-t^{1+p}}}\right)^{-2 /(1-p)} \\
& =(2(1+p))^{1 /(1-p)} B(1 /(1+p), 1 / 2)^{-2 /(1-p)},
\end{aligned}
$$

where $B$ denotes the beta function.

Inequality (2.6) will be proved in Section 4. When $d>0$ is small enough, we can use a general theory in a linear partial differential equation to get a similar estimate to (2.6) in the next remark.

Remark 2.12. We consider the Poisson equation

$$
\left\{\begin{aligned}
-\Delta u=f & \text { in } \Omega, \\
u=0, & \text { on } \partial \Omega .
\end{aligned}\right.
$$

In Gilbarg-Trudinger [5, Theorem 3.7], it is proved that the solution $u(x)$ has a priori bound,

$$
\sup _{\Omega}|u| \leq C \sup _{\Omega}|f|, \quad C=e^{2 d}-1,
$$

provided that $\Omega$ lies between two parallel hyperplanes a distance $2 d$ apart. If $d$ is sufficiently small, then $e^{2 d}-1$ is approximately equal to $2 d$. Let $f=u^{p}$ with $0<p<1$. Then $(2.8)$ means $\|u\|_{\infty} \leq((2+o(1)) d)\|u\|_{\infty}^{p}$, where $o(1)$ converges to zero as $d \rightarrow 0$. This leads to

$$
\|u\|_{\infty} \leq((2+o(1)) d)^{1 /(1-p)} .
$$

Compare this inequality with (2.6). Then (2.9) is not optimal as $d \rightarrow 0$. By using a composite function of $v(t, d)$ and a distance function, we obtain the optimal estimate (2.6).

Example 2.13. A positive solution of

$$
\left\{\begin{aligned}
-\Delta u & =-u \log u, \quad u>0, \quad \text { in } \Omega, \\
u & =0, \quad \text { on } \partial \Omega,
\end{aligned}\right.
$$

has an estimate

$$
\|u(\cdot, \Omega)\|_{\infty} \leq \exp \left(\frac{1}{2}-\frac{\pi^{2}}{4 d^{2}}\right)
$$

The estimates in Examples 2.11 and 2.13 will be computed in Section 4. The above examples deal with $f(s)$ satisfying $f(0)=0$. We consider the case where $f(0)>0$.

Example 2.14. Let $f(s)=e^{-s}, 1-s$ or $(1+s) /(2+s)$. These are examples satisfying (f1)-(f3) and the assumption of Corollary 2.6. Therefore we have (2.1).

\section{Comparison theorem}

In this section, we prove Theorems 2.2, 2.3 and 2.10 . We begin by defining a supersolution and a subsolution. 
Definition 3.1. For $u \in C^{2}(\Omega) \cap C(\bar{\Omega})$ with $u \geq 0$, we call $u$ a classical supersolution of (1.1) if

$$
-\Delta u \geq f(u) \text { in } \Omega .
$$

A classical subsolution is defined by the reverse inequality. We put

$$
C_{0}^{\infty}(\Omega)^{+} \equiv\left\{\phi \in C_{0}^{\infty}(\Omega): \phi \geq 0 \text { in } \Omega\right\} .
$$

For $u \in C(\bar{\Omega})$ with $u \geq 0$, we call $u$ a weak supersolution of (1.1) if

$$
\int_{\Omega}(u \Delta \phi+f(x, u) \phi) d x \leq 0 \quad \text { for } \phi \in C_{0}^{\infty}(\Omega)^{+} .
$$

A weak subsolution is defined by the reverse inequality.

We state two theorems, which are our recent results on the comparison theorem.

Theorem 3.2 ([]). Suppose that (f2) holds. Then the following are equivalent:

(i) $f(s) / s$ is strictly decreasing in $(0, \infty)$.

(ii) Let $\Omega_{0}$ be any bounded open subset of $\mathbb{R}^{N}$ and $u, v$ a positive weak subsolution and a positive weak supersolution in $\Omega_{0}$, respectively. If $u \leq v$ on $\partial \Omega_{0}$, then $u \leq v$ in $\Omega_{0}$. Furthermore, if $u \not \equiv v$ in $\Omega_{0}$, then

$$
\int_{\Omega_{0}}(v f(u)-u f(v)) d x>0 .
$$

(iii) Let $\Omega_{0}$ be any bounded connected open subset of $\mathbb{R}^{N}$, $u$ a positive classical subsolution and $v$ a positive classical supersolution in $\Omega_{0}$. If $u \leq v$ on $\partial \Omega_{0}$, then $u \equiv v$ or $u(x)<v(x)$ in $\Omega_{0}$. In the latter case, (3.1) holds.

Theorem 3.3 ([6]). Suppose that (f1) and (f2) hold. Let $\Omega_{1}$ and $\Omega_{2}$ be bounded open subsets of $\mathbb{R}^{N}$ such that $\Omega_{1} \subset \Omega_{2}, \Omega_{1} \neq \Omega_{2}$ and $\Omega_{2}$ is connected. Let $u_{i}$ be a positive classical solution of (1.1), (1.2) with $\Omega=\Omega_{i}$ for $i=1,2$. Then $u_{1}(x)<u_{2}(x)$ in $\Omega_{1}$.

The theorem above means that the solution becomes larger as the domain is larger. It is known that the comparison theorem is valid for (1.1). See [1] or 4]. However, in many papers, the regularity of $f(s)$ or $\partial \Omega$ is assumed. Our results, Theorems 3.2 and 3.3, need neither the regularity of $f(s)$ nor the smoothness of $\partial \Omega$. By using the theorems above, we prove Theorem 2.10.

Proof of Theorem 2.10. To prove the theorem, it is enough to show that the positive solution is the biggest of all solutions. Suppose that $u$ and $w$ are solutions of (1.1), (1.2) and $u$ is positive but $w$ may change sign. Let $D$ be the set of $x$ where $w(x)>0$. Then Theorem 3.2 means that $w(x) \leq u(x)$ in $D$ and therefore it also holds in $\Omega$. If $f(s)$ is odd, then we have $|w(x)| \leq u(x)$. This completes the proof.

We investigate the properties of the solution $v(t, d)$ of (1.4). Because of (f1) and (f3), $f(s)$ has at most one zero in $(0, \infty)$. We denote it by $s_{0}$ if it exists; otherwise we put $s_{0}=\infty$. If $s_{0}<\infty$, (f1) implies that $f(s)>0$ in $\left(0, s_{0}\right)$ and $f(s)<0$ in $\left(s_{0}, \infty\right)$. If $s_{0}=\infty$, then $f(s)>0$ for all $s>0$.

Lemma 3.4. Let $v(t)=v(t, d)$ be the solution of (1.4). Then $v$ is concave in $(-d, d), v^{\prime}(t)>0$ in $(-d, 0), v^{\prime}(t)<0$ in $(0, d), v(t)$ is even in $(-d, d)$ and $v(t)<s_{0}$ in $(-d, d)$. Moreover, $v(t, d)$ is strictly increasing with respect to $d>0$. The $C^{1}[-d, d]$-norm of $v(t, d)$ converges to zero as $d \rightarrow 0+$. If $f(0)=0$, then the $C^{2}[-d, d]$-norm also converges to zero. 
Proof. By Theorem 1.1, (1.4) has a unique positive solution $v(t)$. Choose $t_{0}$ at which $v(t)$ attains its maximum. If $v\left(t_{0}\right)>s_{0}$, then $-v^{\prime \prime}\left(t_{0}\right)=f\left(v\left(t_{0}\right)\right)<0$. This is impossible. Hence $v(t) \leq s_{0}$ in $(-d, d)$. By Theorem 3.2 (iii), $v(t)<s_{0}$ in $(-d, d)$. Then $-v^{\prime \prime}=f(v)>0$ in $(-d, d)$. Hence $v$ is concave and has a maximum at a unique point $t_{0}$. We show $t_{0}=0$. Suppose on the contrary that $t_{0}>0$. Define $w(t)$ by $w(t)=v(t)$ in $\left[t_{0}, d\right]$ and $w(t)=v\left(2 t_{0}-t\right)$ in $\left[2 t_{0}-d, t_{0}\right]$. Then $w(t)$ is a positive solution of (1.4) in $\left(2 t_{0}-d, d\right)$. Use Theorem 3.3 with $\Omega_{1}=\left(2 t_{0}-d, d\right)$, $\Omega_{2}=(-d, d), u_{1}=w$ and $u_{2}=v$. Then the fact $w \equiv v$ in $\left(t_{0}, d\right)$ contradicts Theorem 3.3. Therefore $t_{0} \leq 0$. In the same way, we find $t_{0} \geq 0$. Thus $t_{0}=0$ and $v^{\prime}(t)>0$ in $(-d, 0), v^{\prime}(t)<0$ in $(0, d)$. Define $w(t)=v(t)$ for $t \geq 0$ and $w(t)=v(-t)$ for $t<0$. Then $w(t)$ is a solution of (1.4). From the uniqueness of solutions, $w$ is identically equal to $v$, i.e., $v$ is even. Theorem 3.3 guarantees that $v(t, d)$ is strictly increasing with respect to $d>0$. We show the convergence of the $C^{1}$-norm of $v(t, d)$. Since $v(t, d) \leq v(0,1)$ for $t \in[-d, d]$ and $0<d<1$, it holds that

$$
\left|v^{\prime \prime}(t, d)\right| \leq \sup _{0 \leq s \leq v(0,1)} f(s) \equiv C .
$$

Integrating over $(0, t)$ and then integrating over $(t, d)$, we have

$$
\left|v^{\prime}(t, d)\right| \leq C t \leq C d, \quad|v(t, d)| \leq C d(d-t) \quad \text { in }[0, d] .
$$

The above inequalities are valid in $[-d, 0]$ also because $v$ is even. Therefore the $C^{1}$-norm of $v$ converges to zero as $d \rightarrow 0+$. If $f(0)=0$, the $C^{2}$-norm also converges to zero because

$$
\left|v^{\prime \prime}(t, d)\right| \leq \sup _{0 \leq s \leq C d^{2}} f(s)
$$

Proof of Theorem 2.2. Since $v\left(x_{N}, d\right)$ with $x=\left(x_{1}, \ldots, x_{N}\right)$ is a solution of (1.1) in $\mathbb{R}^{N-1} \times(-d, d)$ and $v\left(x_{N}, d\right) \geq 0$ on $\partial \Omega$, Theorem 3.2 shows Theorem 2.2.

Proof of Theorem 2.3. Let $\Omega(R, d)$ be as in Theorem 2.3 and let $u(x, R)$ denote the solution of (1.1), (1.2) with $\Omega=\Omega(R, d)$. By Theorems 2.2 and 3.3,

$$
u\left(x, R_{1}\right) \leq u\left(x, R_{2}\right) \leq v\left(x_{N}, d\right) \leq\|v(\cdot, d)\|_{\infty} \quad \text { if } 0<R_{1}<R_{2} .
$$

Hence $\lim _{R \rightarrow \infty} u(x, R)=u_{\infty}(x)$ exists. Fix $R_{0}>0$ arbitrarily. Since

$$
\|\Delta u\|_{\infty}=\|f(u)\|_{\infty} \leq \max _{0 \leq s \leq\|v\|_{\infty}}|f(s)|
$$

the elliptic regularity theorem implies that

$$
\|u(\cdot, R)\|_{2, q, R_{0}} \leq C\left(R_{0}, q\right) \quad \text { for } \quad R>R_{0}+1, q>1 .
$$

Here $\|\cdot\|_{2, q, R_{0}}$ denotes the $W^{2, q}\left(\Omega\left(R_{0}, d\right)\right)$-norm and $C\left(R_{0}, q\right)$ is a constant depending only on $R_{0}$ and $q$. By the Sobolev imbedding theorem and the AscoliArzela theorem, $u(x, R)$ converges to $u_{\infty}(x)$ in $C^{1, \theta}\left(\Omega\left(R_{0}, d\right)\right)$ for any $R_{0}>0$ and $0<\theta<1$. Moreover, $u_{\infty}$ satisfies

$$
\begin{aligned}
-\Delta u_{\infty}=f\left(u_{\infty}\right), & u_{\infty}>0, \quad \text { in } \Omega(\infty, d), \\
u_{\infty}=0, & \text { on } \partial \Omega(\infty, d) .
\end{aligned}
$$


Here $\Omega(\infty, d) \equiv \mathbb{R}^{N-1} \times(-d, d)$. We show that $u_{\infty}\left(x^{\prime}, x_{N}\right)$ is independent of $x^{\prime}$. Let $a \in(-d, d)$ and $x_{0}^{\prime}, y_{0}^{\prime} \in \mathbb{R}^{N-1}$. Put $x_{0}=\left(x_{0}^{\prime}, a\right)$ and $y_{0}=\left(y_{0}^{\prime}, a\right)$. For any $R_{1}$ satisfying $R_{1}>\left|y_{0}^{\prime}\right|$, we define

$$
D\left(R_{1}\right) \equiv\left\{\left(x^{\prime}, x_{N}\right):\left|x^{\prime}+y_{0}^{\prime}-x_{0}^{\prime}\right|<R_{1},\left|x_{N}\right|<d\right\} .
$$

Observe that $u\left(x+y_{0}-x_{0}, R_{1}\right)$ is a solution in $D\left(R_{1}\right)$ and vanishes on $\partial D\left(R_{1}\right)$. Since $D\left(R_{1}\right) \subset \Omega(R, d)$ for $R>0$ large enough, it follows from Theorem 3.3 that

$$
u\left(x+y_{0}-x_{0}, R_{1}\right)<u(x, R) \leq u_{\infty}(x) \quad \text { in } D\left(R_{1}\right) .
$$

Substituting $x=x_{0}$ and letting $R_{1} \rightarrow \infty$, we get $u_{\infty}\left(y_{0}\right) \leq u_{\infty}\left(x_{0}\right)$. By exchanging $x_{0}$ with $y_{0}$, we have the equality. Thus $u_{\infty}\left(x^{\prime}, x_{N}\right)$ is independent of $x^{\prime}$, and it is rewritten as $u_{\infty}\left(x^{\prime}, x_{N}\right)=u_{\infty}\left(x_{N}\right)$. Then we have

$$
\begin{gathered}
-\Delta u_{\infty}=-\partial^{2} u_{\infty} / \partial x_{N}^{2}=f\left(u_{\infty}\right), \quad u_{\infty}>0, \quad \text { in }(-d, d), \\
u_{\infty}(-d)=u_{\infty}(d)=0 .
\end{gathered}
$$

Consequently, $u_{\infty}\left(x_{N}\right)=v\left(x_{N}, d\right)$. If $f(s)$ is locally Hölder continuous, then $u(x, R)$ converges in $C_{l o c}^{2}$ because of the elliptic regularity theorem.

\section{Estimates FOR $v(t, d)$}

In this section, we prove Corollary 2.6 and compute the estimates in Examples 2.11 and 2.13 .

Proof of Corollary 2.6. Since $\sup _{0 \leq s<\infty} f(s) \equiv \bar{f}<\infty$, we have

$$
-v^{\prime \prime}=f(v) \leq \bar{f} \quad \text { in }(-d, d) .
$$

Since $v^{\prime}(0)=0, v(d)=0$ and $v(0)=\|v\|_{\infty}$, we integrate both sides twice to get $\|v\|_{\infty} \leq \bar{f} d^{2} / 2$.

To prove (2.6) and (2.11), we multiply (1.4) by $v^{\prime}(t)$ to get

$$
\frac{d}{d t}\left(\frac{1}{2} v^{\prime}(t)^{2}+F(v)\right)=0, \quad F(v) \equiv \int_{0}^{v} f(s) d s .
$$

Since $v^{\prime}(0)=0$, we have

$$
\frac{1}{2} v^{\prime}(t)^{2}+F(v(t))=\text { const. }=F(v(0)) .
$$

Proof of (2.6). Since $\|u(\cdot, \Omega)\|_{\infty} \leq v(0, d)$, it is enough to prove that $v(0, d)$ satisfies (2.6). By (4.2) with $f(v)=v^{p}$, we obtain

$$
v^{\prime}(t)=-\left[\frac{2}{1+p}\left(M^{1+p}-v(t)^{1+p}\right)\right]^{1 / 2} \quad \text { in }(0, d),
$$

where we have put $M=v(0)=\|v\|_{\infty}$. Dividing both sides by $\left(M^{1+p}-v^{1+p}\right)^{1 / 2}$ and integrating over $(0, d)$, we get

$$
\int_{0}^{M} \frac{1}{\sqrt{M^{1+p}-v^{1+p}}} d v=(2 /(1+p))^{1 / 2} d .
$$

Substituting $v=M u$ in (4.3), we get

$$
M^{(1-p) / 2} \int_{0}^{1} \frac{1}{\sqrt{1-u^{1+p}}} d u=(2 /(1+p))^{1 / 2} d .
$$


This is equivalent to $M=C d^{2 /(1-p)}$, i.e., (2.6) holds, where $C$ is defined by

$$
C=\left(((1+p) / 2)^{1 / 2} \int_{0}^{1} \frac{d u}{\sqrt{1-u^{1+p}}}\right)^{-2 /(1-p)} .
$$

The change of variable $t=u^{1+p}$ yields

$$
\int_{0}^{1} \frac{1}{\sqrt{1-u^{1+p}}} d u=\frac{1}{1+p} B(1 /(1+p), 1 / 2) .
$$

Substituting this relation into (4.5), we obtain (2.7).

Proof of (2.11). By (4.2) with $f(v)=-v \log v$, we obtain

$$
\frac{1}{2} v^{\prime}(t)^{2}-\frac{1}{2} v^{2} \log v+\frac{1}{4} v^{2}=-\frac{1}{2} M^{2} \log M+\frac{1}{4} M^{2}
$$

Rewrite it as

$$
\int_{0}^{M} \frac{d v}{\sqrt{v^{2} \log v-v^{2} / 2-M^{2} \log M+M^{2} / 2}}=d .
$$

Putting $v=M u$, we see

$$
\int_{0}^{1} \frac{d u}{\sqrt{u^{2} \log u+u^{2} \log M-u^{2} / 2-\log M+1 / 2}}=d .
$$

Since $f(v)=-v \log v, s_{0}$ is equal to 1 . Hence $M=\|v\|_{\infty}<1$ and $\log M<0$. We remove $u^{2} \log u$ from (4.6) to get

$$
\int_{0}^{1} \frac{d u}{\sqrt{\left(1-u^{2}\right)(1 / 2-\log M)}} \leq d
$$

or equivalently,

This is reduced to $(2.11)$.

$$
\frac{\pi}{2 d} \leq \sqrt{1 / 2-\log M}
$$

\section{EXTERIOR OF CONVEX DOMAIN}

In this section, we prove Theorem 2.4. To this end, we investigate the properties of the distance function in the lemma below.

Lemma 5.1. Let $D$ be a bounded convex open subset of $\mathbb{R}^{N}$ and define

$$
\rho(x) \equiv \operatorname{dist}(x, \partial D)=\inf \{|x-y|: y \in \partial D\} .
$$

Then $\rho(x)$ is convex in $\mathbb{R}^{N} \backslash \bar{D}$ and is concave in $D$. Also, if $\partial D \in C^{\infty}$, then $\rho \in$ $C^{\infty}\left(\mathbb{R}^{N} \backslash \bar{D}\right)$ and $|\nabla \rho(x)|=1$ in $\mathbb{R}^{N} \backslash \bar{D}$.

Proof. This lemma seems to be known. However, for the sake of completeness, we give a proof. Let $x, y \in \mathbb{R}^{N} \backslash \bar{D}$ and $\xi, \eta \in \bar{D}$ and $0<\theta<1$. Since $\theta \xi+(1-\theta) \eta \in \bar{D}$, we have

$$
\begin{aligned}
\operatorname{dist}(\theta x+(1-\theta) y, \bar{D}) & \leq|\theta x+(1-\theta) y-(\theta \xi+(1-\theta) \eta)| \\
& \leq \theta|x-\xi|+(1-\theta)|y-\eta| .
\end{aligned}
$$

Take the infimum on $\xi$ and $\eta$. Then we get

$$
\operatorname{dist}(\theta x+(1-\theta) y, \bar{D}) \leq \theta \operatorname{dist}(x, \bar{D})+(1-\theta) \operatorname{dist}(y, \bar{D})
$$

Note that $\operatorname{dist}(x, \bar{D})=\operatorname{dist}(x, \partial D)$ in $\mathbb{R}^{N} \backslash \bar{D}$. Then it is convex in $\mathbb{R}^{N} \backslash \bar{D}$. 
We show that $\operatorname{dist}(x, \partial D)$ is concave in $D$. Let $0<\theta<1, x_{i} \in D$ and $r_{i}=$ $\operatorname{dist}\left(x_{i}, \partial D\right)$ for $i=1,2$. Then $B\left(x_{i}, r_{i}\right) \subset D$ for $i=1,2$. Hereafter $B(x, r)$ denotes the open ball centered at $x$ with radius $r$. Let $\xi \in \mathbb{R}^{N}$ with $|\xi|<1$. Then $x_{i}+r_{i} \xi \in D$. This implies

$$
\begin{aligned}
& \theta x_{1}+(1-\theta) x_{2}+\left(\theta r_{1}+(1-\theta) r_{2}\right) \xi \\
& \quad=\theta\left(x_{1}+r_{1} \xi\right)+(1-\theta)\left(x_{2}+r_{2} \xi\right) \in D .
\end{aligned}
$$

Since $\xi(|\xi|<1)$ is arbitrary, we have

$$
B\left(\theta x_{1}+(1-\theta) x_{2}, \theta r_{1}+(1-\theta) r_{2}\right) \subset D,
$$

which means

$$
\begin{aligned}
\operatorname{dist}\left(\theta x_{1}+(1-\theta) x_{2}, \partial D\right) & \geq \theta r_{1}+(1-\theta) r_{2} \\
& =\theta \operatorname{dist}\left(x_{1}, \partial D\right)+(1-\theta) \operatorname{dist}\left(x_{2}, \partial D\right) .
\end{aligned}
$$

Therefore $\rho(x)$ is concave in $D$.

Let $\partial D \in C^{\infty}$. Since $D$ is convex, $\rho$ is smooth in $\mathbb{R}^{N} \backslash \bar{D}$. Fix $x_{0} \in \mathbb{R}^{N} \backslash \bar{D}$ arbitrarily. Since $D$ is convex, there is a unique point $\xi_{0} \in \partial D$ such that $\rho\left(x_{0}\right)=$ $\left|x_{0}-\xi_{0}\right|$. Let $T$ be the tangent space of $\partial D$ at $\xi_{0}$. Since $\rho(x)$ and $|\nabla \rho(x)|$ are invariant under the orthogonal transformation and the parallel translation, we can suppose that $\xi_{0}=0$ and

$$
D \subset\left\{\left(x^{\prime}, x_{N}\right): x_{N}<0\right\}, \quad T=\left\{\left(x^{\prime}, 0\right): x^{\prime} \in \mathbb{R}^{N-1}\right\} .
$$

Then $x_{0}$ is of form $x_{0}=\left(0, \ldots, 0, x_{N}^{0}\right)$ with $x_{N}^{0}>0$. Let $T_{0}$ be a hyperplane parallel to $T$ through $x_{0}$. The restriction $\left.\rho\right|_{T_{0}}$ on $T_{0}$ attains a minimum at $x_{0}$ because $D$ is convex. Therefore $\left(\partial \rho / \partial x_{i}\right)\left(x_{0}\right)=0$ for $1 \leq i \leq n-1$. Since $\rho\left(0, \ldots, 0, x_{N}\right)=x_{N}$ for $x_{N}>0$, we have $\partial \rho\left(0, \ldots, 0, x_{N}\right) / \partial x_{N}=1$. Thus $\left|\nabla \rho\left(x_{0}\right)\right|=1$.

For any bounded convex set, we can make an approximate sequence of smooth convex sets.

Lemma 5.2. Let $D$ be a bounded convex set in $\mathbb{R}^{N}$ and $k$ be a positive integer. Then there exists a bounded convex open set $D_{k}$ with $C^{\infty}$-boundary $\partial D_{k}$ such that

(i) $D \subset D_{k} \subset\left\{x \in \mathbb{R}^{N}: \operatorname{dist}(x, D)<3 / k\right\}$,

(ii) $0 \leq \operatorname{dist}(x, D)-\operatorname{dist}\left(x, D_{k}\right) \leq 3 / k \quad$ for $x \in \mathbb{R}^{N}$,

(iii) $0 \leq \operatorname{dist}\left(x, \mathbb{R}^{N} \backslash D_{k}\right)-\operatorname{dist}\left(x, \mathbb{R}^{N} \backslash D\right) \leq 3 / k \quad$ for $x \in \mathbb{R}^{N}$.

Proof. Choose a function $J \in C_{0}^{\infty}\left(\mathbb{R}^{N}\right)$ such that $J(x) \geq 0$, the support of $J$ is in the unit ball centered at the origin, and the integral of $J(x)$ over $\mathbb{R}^{N}$ is equal to one. For $\varepsilon>0$, we set $J_{\varepsilon}(x)=\varepsilon^{-N} J(x / \varepsilon)$. Put $r(x)=\operatorname{dist}(x, D)$ and define $r_{k}(x) \equiv J_{1 / k} * r(x)$. Here $*$ denotes the convolution. Since $r(x)$ is Lipschitz continuous with Lipschitz constant one, we have

$$
\begin{aligned}
\left|r_{k}(x)-r(x)\right| & \leq \int_{|y| \leq 1 / k} J_{1 / k}(y)|r(x-y)-r(x)| d y \\
& \leq \sup _{|y| \leq 1 / k}|r(x-y)-r(x)| \leq 1 / k .
\end{aligned}
$$

By the Sard theorem (see [7]), the set of all critical values of $r_{k}(x)$ has Lebesgue measure zero. We choose a regular value $\delta_{k} \in(1 / k, 2 / k)$ of $r_{k}(x)$. Set

$$
D_{k} \equiv\left\{x \in \mathbb{R}^{N}: r_{k}(x)<\delta_{k}\right\} .
$$


Since $r(x)$ is convex in $\mathbb{R}^{N}$, so is the mollifier $r_{k}(x)$. Then $D_{k}$ is a convex set. Since $r(x) \rightarrow \infty$ as $|x| \rightarrow \infty, r_{k}(x)$ also diverges to $\infty$ as $|x| \rightarrow \infty$ by (5.2). Therefore $D_{k}$ is bounded. Let $x_{0} \in \partial D_{k}$. Then $r_{k}\left(x_{0}\right)=\delta_{k}$. Since $\delta_{k}$ is a regular value of $r_{k}(x)$, it follows that $\nabla r_{k}\left(x_{0}\right) \neq 0$. By the implicit function theorem, $\partial D_{k}$ belongs to $C^{\infty}$ in a neighborhood of $x_{0}$. Consequently, the whole of $\partial D_{k}$ is a $C^{\infty}$-surface. We show (i). If $x \in D$, then $r(x)=0$. By $(5.2), r_{k}(x) \leq 1 / k<\delta_{k}$, and so $x \in D_{k}$. Thus $D \subset D_{k}$. Let $x \in D_{k}$. By (5.2), we have

$$
r(x) \leq r_{k}(x)+1 / k \leq \delta_{k}+1 / k<3 / k,
$$

which proves the second inclusion in (i).

From $D \subset D_{k}$, it follows that $\operatorname{dist}\left(x, D_{k}\right) \leq \operatorname{dist}(x, D)$. We prove the second inequality in (ii). Let $x \in \mathbb{R}^{N}$. Choose $y \in \overline{D_{k}}$ such that $|x-y|=\operatorname{dist}\left(x, D_{k}\right)$. Then $\operatorname{dist}(y, D) \leq 3 / k$ by (i). Let $\varepsilon>0$ be arbitrary. Then there is a $z \in D$ such that

$$
|y-z|<\operatorname{dist}(y, D)+\varepsilon \leq 3 / k+\varepsilon .
$$

Accordingly, we have

$$
\operatorname{dist}(x, D) \leq|x-z| \leq|x-y|+|y-z| \leq \operatorname{dist}\left(x, D_{k}\right)+3 / k+\varepsilon .
$$

Since $\varepsilon>0$ is arbitrary, this inequality means (ii).

Since $\mathbb{R}^{N} \backslash D_{k} \subset \mathbb{R}^{N} \backslash D$, we have the first inequality in (iii). To prove the second inequality, we show

$$
\operatorname{dist}\left(y, \partial D_{k}\right) \leq 3 / k \text { for } y \in D_{k} \backslash D .
$$

It is enough to show (5.3) for $y \in D_{k} \backslash \bar{D}$. Let $y$ be such a point. Then there is a $z \in \partial D$ such that $\operatorname{dist}(y, D)=|y-z|$. We take a point $\zeta$ on the ray from $z$ through $y$ such that $|\zeta-z|=3 / k$. Note that $r(\zeta)=\operatorname{dist}(\zeta, D)=|\zeta-z|$. Then $r_{k}(\zeta) \geq r(\zeta)-1 / k=2 / k>\delta_{k}$. Since $y \in D_{k}, r_{k}(y)<\delta_{k}$. Therefore there is a point $\eta$ on the segment connecting $y$ with $\zeta$ such that $r_{k}(\eta)=\delta_{k}$, i.e., $\eta \in \partial D_{k}$. Then

$$
\operatorname{dist}\left(y, \partial D_{k}\right) \leq|y-\eta| \leq|z-\zeta|=3 / k
$$

Hence (5.3) holds. We show the second inequality in (iii). We divide $\mathbb{R}^{N}$ into $D$, $D_{k} \backslash D$ and $\mathbb{R}^{N} \backslash D_{k}$. For $x \in \mathbb{R}^{N} \backslash D_{k}$, the inequality is trivial. Let $x \in D$. Then there is a $y \in \partial D$ such that $|x-y|=\operatorname{dist}\left(x, \mathbb{R}^{N} \backslash D\right)$. By (5.3), there is a $z \in \partial D_{k}$ such that $|y-z| \leq 3 / k$. Then

$$
\begin{aligned}
\operatorname{dist}\left(x, \mathbb{R}^{N} \backslash D_{k}\right) & \leq|x-z| \leq|x-y|+|y-z| \\
& \leq \operatorname{dist}\left(x, \mathbb{R}^{N} \backslash D\right)+3 / k .
\end{aligned}
$$

We deal with the case $x \in D_{k} \backslash D$. Then $\operatorname{dist}\left(x, \mathbb{R}^{N} \backslash D\right)=0$. Therefore (5.3) shows the second inequality in (iii).

Proof of Theorem 2.4. For simplicity, we rewrite $v(t, d)$ as $v(t)$.

Step 1. Let $D$ be a bounded convex open set with $C^{\infty}$-boundary.

Put $\rho(x) \equiv \operatorname{dist}(x, \partial D)$. We show that $v(\rho(x))$ is a supersolution in $D_{d}$. Indeed, from an easy calculation it follows that

$$
\Delta v(\rho(x))=v^{\prime \prime}(\rho)|\nabla \rho|^{2}+v^{\prime}(\rho) \Delta \rho .
$$

Note that $|\nabla \rho|=1$ by Lemma 5.1 and $\Delta \rho \geq 0$ because $\rho(x)$ is convex in $D_{d}$. Since $0<\rho(x)<d$ in $D_{d}, v^{\prime}(\rho(x))<0$ in $D_{d}$ because of Lemma 3.4. Since $v(t)$ is a 
solution of $(1.4),(5.4)$ leads to

$$
-\Delta v(\rho) \geq f(v(\rho)) .
$$

Thus $v(\rho(x))$ is a classical supersolution. By Theorem 3.2, $u(x, \Omega) \leq v(\rho(x))$ in $\Omega$.

Step 2 . Let $D$ be any bounded convex set in $\mathbb{R}^{N}$.

Define $D_{k}$ by Lemma 5.2 and put

$$
R_{k}(x)=\operatorname{dist}\left(x, D_{k}\right), \quad R(x)=\operatorname{dist}(x, D) .
$$

Note that $R(x)=\rho(x)$ in $\mathbb{R}^{N} \backslash D$. By Lemma 5.2 (ii), $R_{k}(x)$ uniformly converges to $R(x)$ on $\mathbb{R}^{N}$. We show that $v(R(x))$ is a weak supersolution. Let $\phi \in C_{0}^{\infty}\left(D_{d}\right)^{+}$. Then the support of $\phi$ is contained in $\left(D_{k}\right)_{d}$ for $k$ large enough, where

$$
\left(D_{k}\right)_{d} \equiv\left\{x \in \mathbb{R}^{N} \backslash \bar{D}_{k}: \operatorname{dist}\left(x, D_{k}\right)<d\right\} .
$$

Since $v\left(R_{k}(x)\right)$ is a supersolution in $\left(D_{k}\right)_{d}$ by Step 1, we have

$$
\int_{\left(D_{k}\right)_{d}}\left\{v\left(R_{k}(x)\right) \Delta \phi+f\left(v\left(R_{k}(x)\right)\right) \phi\right\} d x \leq 0 .
$$

Letting $k \rightarrow \infty$, we have

$$
\int_{D_{d}}\{v(R(x)) \Delta \phi+f(v(R(x))) \phi\} d x \leq 0 .
$$

Accordingly, $v(R(x))=v(\rho(x))$ is a weak supersolution.

\section{INTERIOR OF CONVEX DOMAIN}

In this section, we prove Theorem 2.5. Even if $\Omega$ is not necessarily convex, we call $u$ a concave function in $\Omega$ if for any segment $L$ in $\Omega$, the restriction of $u$ on $L$ is concave.

Lemma 6.1. Let $\Omega$ be a bounded open subset of $\mathbb{R}^{N}$ with smooth boundary. Let $u$ be continuous and concave in $\Omega_{\delta}$ with a small $\delta>0$, where

$$
\Omega_{\delta} \equiv\left\{x \in \mathbb{R}^{N}: \operatorname{dist}(x, \Omega)<\delta\right\} .
$$

Moreover, suppose that $u$ is of class $C^{1}$ in a neighborhood of $\partial \Omega$. If $\phi \in C^{2}(\bar{\Omega})$ and $\phi \geq 0$ in $\Omega$, then

$$
\int_{\partial \Omega}\left(u \frac{\partial \phi}{\partial \nu}-\phi \frac{\partial u}{\partial \nu}\right) d s \geq \int_{\Omega} u \Delta \phi d x .
$$

Here $\partial / \partial \nu$ denotes the outward normal derivative.

Proof. We use a mollifier $u_{\varepsilon} \equiv J_{\varepsilon} * u$, where $J_{\varepsilon}$ is defined in the proof of Lemma 5.2. Since $u$ is concave in $\Omega_{\delta}$, so is $u_{\varepsilon}$ in $\Omega$ for $0<\varepsilon<\delta$. Then $\Delta u_{\varepsilon} \leq 0$ in $\Omega$. Therefore the Green formula gives

$$
\int_{\partial \Omega}\left(u_{\varepsilon} \frac{\partial \phi}{\partial \nu}-\phi \frac{\partial u_{\varepsilon}}{\partial \nu}\right) d s=\int_{\Omega}\left(u_{\varepsilon} \Delta \phi-\phi \Delta u_{\varepsilon}\right) d x \geq \int_{\Omega} u_{\varepsilon} \Delta \phi d x .
$$

Letting $\varepsilon \rightarrow 0$, we have (6.1).

The next lemma and Theorem 6.3 play the most important roles in the proof of Theorem 2.5. We denote the $\mathbb{R}^{N}$-Lebesgue measure by $\operatorname{vol}(\cdot)$. 
Lemma 6.2. Let $D$ be a bounded open subset of $\mathbb{R}^{N}$ and $K$ a compact subset of $\mathbb{R}^{N}$ such that $\operatorname{vol}(K)=0$ and $K \subset D$. Suppose that $w \in C(\bar{D}) \cap C^{2}(D \backslash K)$, w is non-negative and concave in $D$ and

$$
-\Delta w \geq f(w) \text { in } D \backslash K
$$

Then $w$ is a weak supersolution in $D$.

Proof. Let $\varepsilon>0$. Since $K$ is compact and $\operatorname{vol}(K)=0$, there is an open subset $\Omega(\varepsilon)$ such that $\operatorname{vol}(\Omega(\varepsilon))<\varepsilon, \partial \Omega(\varepsilon)$ is smooth and

$$
K \subset \Omega(\varepsilon) \subset \overline{\Omega(\varepsilon)} \subset D .
$$

We denote the $L^{2}(\Omega)$-inner product by $(\cdot, \cdot)_{\Omega}$. Let $\phi \in C_{0}^{\infty}(D)^{+}$. By $(6.2)$, we have

$$
\begin{aligned}
& (w,-\Delta \phi)_{D \backslash \Omega(\varepsilon)}\left(\phi \frac{\partial w}{\partial \nu}-w \frac{\partial \phi}{\partial \nu}\right) d s+(-\Delta w, \phi)_{D \backslash \Omega(\varepsilon)} \\
& =\int_{\partial(D \backslash \Omega(\varepsilon))}\left(\phi \frac{\partial w}{\partial \nu}-w \frac{\partial \phi}{\partial \nu}\right) d s+(f(w), \phi)_{D \backslash \Omega(\varepsilon)} .
\end{aligned}
$$

Since $\phi$ vanishes near $\partial D$, we use Lemma 6.1 to get

$$
\begin{aligned}
\int_{\partial(D \backslash \Omega(\varepsilon))}\left(\phi \frac{\partial w}{\partial \nu}-w \frac{\partial \phi}{\partial \nu}\right) d s & =-\int_{\partial \Omega(\varepsilon)}\left(\phi \frac{\partial w}{\partial \nu}-w \frac{\partial \phi}{\partial \nu}\right) d s \\
& \geq \int_{\Omega(\varepsilon)} w \Delta \phi d x \\
& \geq-\varepsilon\|w\|_{L^{\infty}(D)}\|\Delta \phi\|_{L^{\infty}(D)},
\end{aligned}
$$

where we have used the fact that $\operatorname{vol}(\Omega(\varepsilon)) \leq \varepsilon$. Then (6.3) is reduced to

$$
(w,-\Delta \phi)_{D \backslash \Omega(\varepsilon)} \geq-\varepsilon\|w\|_{L^{\infty}(D)}\|\Delta \phi\|_{L^{\infty}(D)}+(f(w), \phi)_{D \backslash \Omega(\varepsilon)} .
$$

Letting $\varepsilon \rightarrow 0$, we have

$$
(w,-\Delta \phi)_{D} \geq(f(w), \phi)_{D}
$$

Thus $w$ is a weak supersolution in $D$.

To prove Theorem 2.5, we need the next theorem, which will be proved in Section 7 .

Theorem 6.3. Let $D$ be a bounded open convex subset of $\mathbb{R}^{N}$ with $C^{\infty}$-boundary and put $\rho(x) \equiv \operatorname{dist}(x, \partial D)$. Then there exists a subset $K$ of $D$ such that $K$ is compact, $\operatorname{vol}(K)=0, \rho \in C^{\infty}(D \backslash K)$ and $|\nabla \rho(x)|=1$ in $D \backslash K$.

Proof of Theorem 2.5. We have only to prove that $v(\rho(x)-d)$ is a weak supersolution in $D$.

Step 1 . Let $D$ be a bounded convex open set with $C^{\infty}$-boundary.

Let $K$ be defined by Theorem 6.3. Observe the identity,

$$
\Delta v(\rho(x)-d)=v^{\prime \prime}(\rho-d)|\nabla \rho|^{2}+v^{\prime}(\rho-d) \Delta \rho .
$$

Since $\rho$ is concave in $D, \Delta \rho \leq 0$ in $D \backslash K$. Since $d$ is the radius of the maximum ball in $D$, we have $0<\rho(x) \leq d$ in $D$. Then $v^{\prime}(\rho(x)-d) \geq 0$ in $D$ because of Lemma 3.4. Since $v(t)$ is a solution of (1.4) and $|\nabla \rho|=1$ in $D \backslash K$, we obtain

$$
-\Delta v(\rho-d) \geq f(v(\rho-d)) \quad \text { in } D \backslash K \text {. }
$$


Since $v(t)$ is concave and increasing in $(-d, 0)$ and $\rho(x)$ is concave in $D, v(\rho(x)-d)$ is also concave in $D$. Then by Lemma $6.2, v(\rho(x)-d)$ becomes a weak supersolution in $D$.

Step 2. Let $D$ be bounded open and convex.

Define $D_{k}$ by Lemma 5.2 and put

$$
R_{k}(x)=\operatorname{dist}\left(x, \mathbb{R}^{N} \backslash D_{k}\right), \quad R(x)=\operatorname{dist}\left(x, \mathbb{R}^{N} \backslash D\right) .
$$

Note that $R(x)=\rho(x)$ in $D$. By Lemma 5.2 (iii), $R_{k}(x)$ uniformly converges to $R(x)$ on $\mathbb{R}^{N}$. Let $d_{k}$ denote the radius of the maximum ball in $D_{k}$. By Step 1, $v\left(R_{k}(x)-d_{k}, d_{k}\right)$ is a weak supersolution in $D_{k}$. Letting $k \rightarrow \infty$, we verify that $v(R(x)-d, d)$ is also a weak supersolution in $D$.

\section{Singular SET IN CONVEX DOMAIN}

In this section, we prove Theorem 6.3. Before going to the proof, we observe how $K$ appears in $D$. Here $K$ is the set of points at which $\operatorname{dist}(x, \partial D)$ is not differentiable. If $D$ is a ball, then $K$ consists only of the center of $D$. If $D=$ $(-a, a) \times(-b, b)$ is a rectangular in $\mathbb{R}^{2}$ with $0<b<a$, then

$$
\begin{aligned}
& \rho(x, y)=\operatorname{dist}((x, y), \partial D)=\min (a-|x|, b-|y|), \\
& K=\{(x, y) \in D: a-|x|=b-|y|\} \\
& \cup\{(x, y) \in D:|x| \leq a-b, y=0\} .
\end{aligned}
$$

Observing $K$ above, we see that $\rho(x)$ is not differentiable at a point $x_{0}$ if the maximum sphere centered at $x_{0}$ that is contained in $\bar{D}$ is tangent to $\partial D$ at two or more points. In other words, for $\xi \in \partial D$, let $B(\xi)$ be the maximum ball such that $B \subset D$ and $\xi \in \partial B \cap \partial D$. Denote the center of $B(\xi)$ by $p(\xi)$. Then the mapping $p$ is well-defined and $\rho(x)$ is differentiable in $D \backslash p(\partial D)$. Our proof of Theorem 6.3 is based on this point of view. For any domain $D, K$ is always non-empty because it includes the center of the maximum ball in $D$. To prove Theorem 6.3 , we need a notion of principal curvatures. Throughout this section, we suppose that $D$ is a bounded convex open set in $\mathbb{R}^{N}$ with $C^{\infty}$-boundary and denote by $n(x)$ the inward unit normal vector at $x \in \partial D$ and by $\Lambda(x)$ the maximum of the principal curvatures at $x$. The definition of $\Lambda(x)$ will be given exactly in the proof of the next lemma.

Lemma 7.1. $\Lambda(x)$ is Lipschitz continuous on $\partial D$.

Proof. Let $x_{0} \in \partial D$ and $T$ be the tangent space at $x_{0}$. Since the principal curvatures are invariant under the parallel translation and the orthogonal transformation, we choose $x_{0}=0$ and

$$
\begin{gathered}
D \subset\left\{\left(x^{\prime}, x_{N}\right): x^{\prime} \in \mathbb{R}^{N-1}, x_{N}>0\right\}, \\
T=\left\{\left(x^{\prime}, 0\right): x^{\prime} \in \mathbb{R}^{N-1}\right\} .
\end{gathered}
$$

Since $\partial D$ is smooth, it is locally represented as a graph of a certain function $\phi(\cdot)$, i.e., there are $\varepsilon>0$ and a neighborhood $U$ of the origin such that

$$
\partial D \cap U=\left\{\left(x^{\prime}, \phi\left(x^{\prime}\right)\right): x^{\prime} \in B_{N-1}(0, \varepsilon)\right\},
$$

where $x^{\prime}=\left(x_{1}, \ldots, x_{N-1}\right) \in \mathbb{R}^{N-1}$ and $B_{N-1}(0, \varepsilon)$ denotes a ball in $\mathbb{R}^{N-1}$ centered at the origin with radius $\varepsilon$. By (7.1), we have

$$
\phi\left(x^{\prime}\right) \geq 0, \quad \phi(0, \ldots, 0)=0, \quad \nabla \phi(0, \ldots, 0)=(0, \ldots, 0) .
$$


For $x^{\prime}=\left(x_{1}, \ldots, x_{N-1}\right) \in B_{N-1}(0, \varepsilon)$, we put

$$
u\left(x^{\prime}\right)=\left(x_{1}, \ldots, x_{N-1}, \phi\left(x_{1}, \ldots, x_{N-1}\right)\right) .
$$

We denote the inner product of vectors $x, y \in \mathbb{R}^{N}$ by $(x, y)$. We define the $(N-1) \times$ $(N-1)$ matrix $G\left(x^{\prime}\right)$ whose $(i, j)$-th element is the inner product $\left(\partial u / \partial x_{i}, \partial u / \partial x_{j}\right)$, that is,

$$
G\left(x^{\prime}\right)=\left(\phi_{x_{i}}\left(x^{\prime}\right) \phi_{x_{j}}\left(x^{\prime}\right)+\delta_{i j}\right)_{1 \leq i, j \leq N-1} .
$$

Here $\delta_{i j}$ stands for Kronecker's symbol, i.e., $\delta_{i j}=1$ if $i=j$ and $\delta_{i j}=0$ if $i \neq j$. We denote the inward unit normal vector at $u\left(x^{\prime}\right) \in \partial D$ by $n\left(x^{\prime}\right)$. Then it is represented as

$$
n\left(x^{\prime}\right)=\frac{1}{\sqrt{\left|\nabla \phi\left(x^{\prime}\right)\right|^{2}+1}}\left(-\phi_{x_{1}}, \ldots,-\phi_{x_{N-1}}, 1\right) .
$$

We define the $(N-1) \times(N-1)$ matrix $H\left(x^{\prime}\right)$ whose $(i, j)$-th element is the inner product $\left(\partial^{2} u / \partial x_{i} \partial x_{j}, n\left(x^{\prime}\right)\right)$, that is,

$$
H\left(x^{\prime}\right)=\frac{1}{\sqrt{\left|\nabla \phi\left(x^{\prime}\right)\right|^{2}+1}}\left(\phi_{x_{i} x_{j}}\left(x^{\prime}\right)\right)_{1 \leq i, j \leq N-1} .
$$

Then $G$ and $H$ are symmetric matrices and $G$ is positive definite. Indeed, for $\xi={ }^{t}\left(\xi_{1}, \ldots, \xi_{N-1}\right)$, we have

$$
\left(G\left(x^{\prime}\right) \xi, \xi\right)=\sum_{i, j}\left(\phi_{x_{i}} \phi_{x_{j}}+\delta_{i j}\right) \xi_{i} \xi_{j}=\left(\sum_{i} \phi_{x_{i}} \xi_{i}\right)^{2}+|\xi|^{2} \geq|\xi|^{2}
$$

where $|\xi|=\left(\xi_{1}^{2}+\cdots+\xi_{N-1}^{2}\right)^{1 / 2}$. Since $D$ is convex, $\phi\left(x^{\prime}\right)$ is a convex function and therefore the Hessian matrix $H\left(x^{\prime}\right)$ is non-negative. Then $G\left(x^{\prime}\right)^{-1} H\left(x^{\prime}\right)$ is a nonnegative symmetric matrix and has non-negative eigenvalues $\lambda_{i}\left(x^{\prime}\right)$ with $1 \leq i \leq$ $N-1$. They are called principal curvatures. They do not depend on the choice of the coordinate system. We denote the maximum of principal curvatures by $\Lambda\left(x^{\prime}\right)$, i.e., $\Lambda\left(x^{\prime}\right)=\max _{1 \leq i \leq N-1} \lambda_{i}\left(x^{\prime}\right)$. Put $F\left(x^{\prime}\right)=G\left(x^{\prime}\right)^{-1} H\left(x^{\prime}\right)$. Since $\Lambda\left(x^{\prime}\right)$ is the maximum eigenvalue of $F\left(x^{\prime}\right)$, we have

$$
\Lambda\left(x^{\prime}\right) \equiv \max _{|\xi|=1}\left(F\left(x^{\prime}\right) \xi, \xi\right)
$$

Since $G\left(x^{\prime}\right)$ and $H\left(x^{\prime}\right)$ are smooth, $F\left(x^{\prime}\right)$ is Lipschitz continuous. Then $\Lambda\left(x^{\prime}\right)$ is also Lipschitz continuous on $\overline{B_{N-1}(0, \varepsilon)}$ by (7.8). Since $x_{0} \in \partial D$ is arbitrary and $\partial D$ is compact, $\Lambda(\cdot)$ is Lipschitz continuous on $\partial D$.

We need another characterization of $\Lambda(x)$, which is as follows. Let $x \in \partial D$ and denote the tangent space at $x$ by $T_{x}$ and the inward unit normal vector by $n(x)$. Let $v \in T_{x} \backslash\{0\}$ be any non-zero tangent vector. We define $P(x, v)$ by the plane that is spanned by $n(x)$ and $v$ and contains $x$, i.e.,

$$
P(x, v) \equiv\{x+\operatorname{sn}(x)+t v: s, t \in \mathbb{R}\} .
$$

Then $P(x, v) \cap \partial D$ forms a plane curve. We denote its curvature at $x$ by $\Lambda(x, v)$. This is called a normal curvature toward $v$. Then $\Lambda(x)$ is characterized as

$$
\Lambda(x) \equiv \max _{v \in T_{x} \backslash\{0\}} \Lambda(x, v) .
$$


The reciprocals $1 / \lambda_{1}(x), \ldots, 1 / \lambda_{N-1}(x)$ of the principal curvatures are called the radii of principal curvatures. We denote the minimum of them by $R(x)$, i.e.,

$$
R(x) \equiv \begin{cases}\frac{1}{\Lambda(x)} & \text { if } \quad \Lambda(x)>0, \\ \infty & \text { if } \quad \Lambda(x)=0 .\end{cases}
$$

From Lemma 7.1, the next lemma follows.

Lemma 7.2. For any $a>0, R(x)$ is Lipschitz continuous on the set of $x \in \partial D$ satisfying $R(x) \leq a$.

Recall that $n(x)$ denotes the inward unit normal vector at $x \in \partial D$. Then the ball with radius $r$ which is internally tangent to $\partial D$ at $x$ is represented as $B(x+r n(x), r)$. If $r<R(x)$, then $B(x+r n(x), r) \cap B(x, \delta) \subset D$ for $\delta>0$ small enough. If $r>R(x)$, then $B(x+r n(x), r)$ cannot be inscribed in $D$. We can take $\delta>0$ locally uniformly on $x \in \partial D$. More precisely, we have the next lemma.

Lemma 7.3. Let $x_{0} \in \partial D$. Then the following assertions hold:

(i) For any $r_{0} \in\left(0, R\left(x_{0}\right)\right)$, there exists a $\delta>0$ such that

$$
B\left(x+r_{0} n(x), r_{0}\right) \cap B(x, \delta) \subset D \quad \text { for } x \in B\left(x_{0}, \delta\right) \cap \partial D .
$$

(ii) For any $r_{0}>R\left(x_{0}\right)$, there exists a $\delta>0$ such that

$$
\left(B\left(x+r_{0} n(x), r_{0}\right) \cap B(x, \varepsilon)\right) \backslash \bar{D} \neq \emptyset,
$$

for $x \in B\left(x_{0}, \delta\right) \cap \partial D$ and any $\varepsilon>0$.

Proof. We show (i). Since $\partial D$ is smooth, we have a $\delta_{0}>0$ such that

$$
x+r n(x) \in D \quad \text { for } r \in\left(0, \delta_{0}\right) \text { and } x \in \partial D .
$$

Let $x_{0} \in \partial D$ and $0<r_{0}<R\left(x_{0}\right)$. Then we choose $\delta \in\left(0, \delta_{0}\right)$ so small that

$$
r_{0}<R(x) \text { for } x \in \overline{B\left(x_{0}, 2 \delta\right)} \cap \partial D \text {. }
$$

Let $x \in B\left(x_{0}, \delta\right) \cap \partial D, v \in T_{x} \backslash\{0\}$ and define $P(x, v)$ by (7.9). We introduce the coordinate system $(t, s)$ on $P(x, v)$. Choose the $v$-direction as the $t$-axis, the $n(x)$ direction as the $s$-axis, and the point $x$ as the origin. Then $\partial D \cap P(x, v) \cap B(x, \delta)$ forms a curve, say $C_{1}$, which is a graph of a certain function $s=g(t)$. Note that $g(0)=0, g(t) \geq 0$ and it is convex. On the other hand, $\partial B\left(x+r_{0} n(x), r_{0}\right) \cap P(x, v)$ becomes a circle $C_{2}$,

$$
C_{2}: t^{2}+\left(s-r_{0}\right)^{2}=r_{0}^{2} .
$$

Then $C_{1}$ and $C_{2}$ are tangent at $(t, s)=(0,0)$. (7.13) means that the curvature $1 / r_{0}$ of $C_{2}$ is greater than the maximum of curvatures of all points $x$ on $C_{1}$. Therefore the graph of $C_{2}$ lies over the graph of $C_{1}$. This fact with (7.12) shows

$$
B\left(x+r_{0} n(x), r_{0}\right) \cap P(x, v) \cap B(x, \delta) \subset D .
$$

Since $v \in T_{x} \backslash\{0\}$ is arbitrary, the inclusion above implies the assertion (i).

We show (ii). Let $r_{0}>R\left(x_{0}\right)$. Choose $\delta>0$ such that $r_{0}>R(x)$ for $x \in$ $B\left(x_{0}, \delta\right) \cap \partial D$. Let $x \in B\left(x_{0}, \delta\right) \cap \partial D$. We choose $v \in T_{x} \backslash\{0\}$ that attains the maximum in (7.10), i.e., $\Lambda(x)=\Lambda(x, v)$. Let $C_{1}$ and $C_{2}$ be as in the proof of (i). Since the curvature of $C_{1}$ at $(t, s)=(0,0)$ is greater than that of $C_{2}$, the graph of $C_{1}$ lies over that of $C_{2}$ in a small neighborhood of the origin. This means

$$
\left(B\left(x+r_{0} n(x), r_{0}\right) \cap P(x, v) \cap B(x, \varepsilon)\right) \backslash(\bar{D} \cap P(x, v)) \neq \emptyset,
$$


for any $\varepsilon>0$, which is rewritten as

$$
\left(B\left(x+r_{0} n(x), r_{0}\right) \cap B(x, \varepsilon) \backslash \bar{D}\right) \cap P(x, v) \neq \emptyset .
$$

This means the assertion (ii).

To prove Theorem 6.3, we define $r(x)$ and $p(x)$ as follows. Let $x \in \partial D$. Take the maximum ball $B$ that is internally tangent to $\partial D$ at $x$ and $B \subset D$. Then we define $r(x)$ and $p(x)$ by the radius and the center of $B$, respectively. In other words, we put

$$
\begin{gathered}
r(x) \equiv \max \{r>0: B(x+r n(x), r) \subset D\}, \\
p(x) \equiv x+r(x) n(x) .
\end{gathered}
$$

Note that $r(x) \leq \min (R(x), d)$ on $\partial D$ because of Lemma 7.3. Recall that $d$ denotes the radius of the maximum ball in $D$.

Lemma 7.4. $p(x)$ and $r(x)$ are continuous on $\partial D$.

Proof. We show that $r(x)$ is continuous. Once it is proved, $p(x)$ is also continuous by $(7.15)$. Let $x_{k}, x_{0} \in \partial D$ and $\left\{x_{k}\right\}$ converge to $x_{0}$.

Step 1. We show that $\limsup _{k \rightarrow \infty} r\left(x_{k}\right) \leq r\left(x_{0}\right)$.

Fix $a \in\left(r\left(x_{0}\right), \infty\right)$ arbitrarily. Suppose that $B\left(x_{k}^{\prime}+a n\left(x_{k}^{\prime}\right), a\right) \subset D$ with a subsequence $\left\{x_{k}^{\prime}\right\}$ of $\left\{x_{k}\right\}$. Then $B\left(x_{0}+a n\left(x_{0}\right), a\right) \subset D$. This contradicts the fact that $r\left(x_{0}\right)<a$. Therefore $B\left(x_{k}+a n\left(x_{k}\right), a\right) \not \subset D$ for $k$ large enough. This means that $r\left(x_{k}\right)<a$, and so $\lim \sup _{k \rightarrow \infty} r\left(x_{k}\right) \leq a$. Since $a \in\left(r\left(x_{0}\right), \infty\right)$ is arbitrary, we conclude that $\limsup _{k \rightarrow \infty} r\left(x_{k}\right) \leq r\left(x_{0}\right)$.

Step 2. We show that $r\left(x_{0}\right) \leq \liminf _{k \rightarrow \infty} r\left(x_{k}\right)$.

Fix $a \in\left(0, r\left(x_{0}\right)\right)$ arbitrarily. Since $r\left(x_{0}\right) \leq R\left(x_{0}\right)$, we choose a $\delta>0$ by Lemma 7.3 (i) such that

$$
B(x+a n(x), a) \cap B(x, \delta) \subset D,
$$

for $x \in B\left(x_{0}, \delta\right) \cap \partial D$. We show that there exists an $\varepsilon \in(0, \delta)$ such that

$$
B(x+a n(x), a) \backslash B(x, \delta) \subset D,
$$

for $x \in B\left(x_{0}, \varepsilon\right) \cap \partial D$. Suppose that this is false. Then there exist sequences $\left\{y_{k}\right\}$ and $\left\{z_{k}\right\}$ such that $y_{k} \in \partial D,\left\{y_{k}\right\}$ converges to $x_{0}$ and

$$
\left|y_{k}+a n\left(y_{k}\right)-z_{k}\right|<a, \quad\left|z_{k}-y_{k}\right| \geq \delta, \quad z_{k} \notin D \text {. }
$$

We choose a convergent subsequence of $\left\{z_{k}\right\}$ and denote its limit by $z_{0}$. Then we have

$$
\left|x_{0}+a n\left(x_{0}\right)-z_{0}\right| \leq a, \quad\left|z_{0}-x_{0}\right| \geq \delta, \quad z_{0} \notin D .
$$

This contradicts the fact that

$$
\overline{B\left(x_{0}+a n\left(x_{0}\right), a\right)} \backslash\left\{x_{0}\right\} \subset B\left(x_{0}+r\left(x_{0}\right) n\left(x_{0}\right), r\left(x_{0}\right)\right) \subset D .
$$

Accordingly, (7.17) holds. By (7.16) and (7.17), $B(x+a n(x), a)$ is a subset of $D$ for $x \in B\left(x_{0}, \varepsilon\right) \cap \partial D$. Hence $B\left(x_{k}+a n\left(x_{k}\right), a\right) \subset D$ for $k$ large enough. Therefore $a \leq r\left(x_{k}\right)$ and $a \leq \liminf _{k \rightarrow \infty} r\left(x_{k}\right)$. Since $a \in\left(0, r\left(x_{0}\right)\right)$ is arbitrary, we obtain the claim of Step 2. Steps 1 and 2 complete the proof. 
Since $n(x)$ is smooth on $\partial D$, it is Lipschitz continuous. Denote the Lipschitz constant by $L$, and we have

$$
|n(x)-n(y)| \leq L|x-y| \quad \text { for } x, y \in \partial D .
$$

For each $a>0$, we define

$$
S_{a} \equiv\{x \in \partial D: r(x) \leq R(x)-a\} .
$$

Lemma 7.5. For any $a>0, p(x)$ and $r(x)$ are Lipschitz continuous on $S_{a}$ if it is non-empty.

To prove the lemma above, we use a contradiction. Suppose that $p(x)$ is not Lipschitz continuous on $S_{a}$. Then there exist sequences $\left\{x_{k}\right\}$ and $\left\{y_{k}\right\}$ on $S_{a}$ such that

$$
\lim _{k \rightarrow \infty} \frac{\left|p\left(y_{k}\right)-p\left(x_{k}\right)\right|}{\left|y_{k}-x_{k}\right|}=\infty .
$$

We can assume that $r\left(x_{k}\right) \leq r\left(y_{k}\right)$ because we exchange $x_{k}$ with $y_{k}$ for each $k$ if necessary. If $r\left(x_{k}\right)=r\left(y_{k}\right)$ with infinitely many $k$, then we use (7.15) and (7.18) to get

$$
\begin{aligned}
\left|p\left(y_{k}\right)-p\left(x_{k}\right)\right| & \leq\left|y_{k}-x_{k}\right|+r\left(x_{k}\right)\left|n\left(y_{k}\right)-n\left(x_{k}\right)\right| \\
& \leq\left|y_{k}-x_{k}\right|+d L\left|y_{k}-x_{k}\right| .
\end{aligned}
$$

This contradicts (7.20). Therefore $r\left(x_{k}\right)<r\left(y_{k}\right)$ for $k$ large enough. By (7.20), $\left|x_{k}-y_{k}\right|$ converges to zero. We choose subsequences (again denoted by $\left\{x_{k}\right\}$ and $\left.\left\{y_{k}\right\}\right)$ of $\left\{x_{k}\right\}$ and $\left\{y_{k}\right\}$ which converge to a common limit $x_{0}$. Accordingly, $\left\{x_{k}\right\}$ and $\left\{y_{k}\right\}$ satisfy the next assumption.

Assumption 7.6. $\left\{x_{k}\right\},\left\{y_{k}\right\} \subset S_{a}, r\left(x_{k}\right)<r\left(y_{k}\right),\left\{x_{k}\right\}$ and $\left\{y_{k}\right\}$ converge to a common limit $x_{0} \in S_{a}$, and (7.20) holds.

Lemma 7.7. Under Assumption 7.6, $\left(p\left(y_{k}\right)-p\left(x_{k}\right)\right) /\left|p\left(y_{k}\right)-p\left(x_{k}\right)\right|$ converges to $n\left(x_{0}\right)$.

Proof. By (7.15), we have

$$
p\left(y_{k}\right)-p\left(x_{k}\right)=r\left(y_{k}\right) n\left(y_{k}\right)-r\left(x_{k}\right) n\left(x_{k}\right)+y_{k}-x_{k} .
$$

Taking the inner product of (7.21) and $n\left(x_{k}\right)$ and using (7.18), we get

$$
\begin{aligned}
& \left(p\left(y_{k}\right)-p\left(x_{k}\right), n\left(x_{k}\right)\right) \\
& \quad=\left(r\left(y_{k}\right)-r\left(x_{k}\right)\right)\left(n\left(y_{k}\right), n\left(x_{k}\right)\right) \\
& \quad+r\left(x_{k}\right)\left(n\left(y_{k}\right)-n\left(x_{k}\right), n\left(x_{k}\right)\right)+\left(y_{k}-x_{k}, n\left(x_{k}\right)\right) \\
& \geq\left(r\left(y_{k}\right)-r\left(x_{k}\right)\right)\left(n\left(y_{k}\right), n\left(x_{k}\right)\right)-(d L+1)\left|y_{k}-x_{k}\right| .
\end{aligned}
$$

On the other hand, we estimate (7.21) as

$$
\begin{aligned}
& \left|p\left(y_{k}\right)-p\left(x_{k}\right)\right| \\
& \quad \leq\left(r\left(y_{k}\right)-r\left(x_{k}\right)\right)\left|n\left(y_{k}\right)\right|+r\left(x_{k}\right)\left|n\left(y_{k}\right)-n\left(x_{k}\right)\right|+\left|y_{k}-x_{k}\right| \\
& \quad \leq r\left(y_{k}\right)-r\left(x_{k}\right)+(d L+1)\left|y_{k}-x_{k}\right| .
\end{aligned}
$$

Dividing both sides by $\left|p\left(y_{k}\right)-p\left(x_{k}\right)\right|$, letting $k \rightarrow \infty$ and using (7.20), we get

$$
1 \leq \liminf _{k \rightarrow \infty} \frac{r\left(y_{k}\right)-r\left(x_{k}\right)}{\left|p\left(y_{k}\right)-p\left(x_{k}\right)\right|} .
$$


Put $q_{k}=\left(p\left(y_{k}\right)-p\left(x_{k}\right)\right) /\left|p\left(y_{k}\right)-p\left(x_{k}\right)\right|$. Dividing (7.22) by $\left|p\left(y_{k}\right)-p\left(x_{k}\right)\right|$ and letting $k \rightarrow \infty$, we have

$$
\liminf _{k \rightarrow \infty}\left(q_{k}, n\left(x_{k}\right)\right) \geq \liminf _{k \rightarrow \infty} \frac{r\left(y_{k}\right)-r\left(x_{k}\right)}{\left|p\left(y_{k}\right)-p\left(x_{k}\right)\right|}\left(n\left(y_{k}\right), n\left(x_{k}\right)\right) \geq 1,
$$

because $\left\{x_{k}\right\}$ and $\left\{y_{k}\right\}$ converge to $x_{0}$. Since $\left|q_{k}\right|=1,(7.25)$ implies that $\left(q_{k}, n\left(x_{k}\right)\right)$ converges to 1 . Consequently, $\left\{q_{k}\right\}$ converges to $n\left(x_{0}\right)$.

Lemma 7.8. Impose Assumption 7.6. Then for any $\varepsilon>0$ there exists a $k_{0} \in \mathbb{N}$ such that

$$
\left(\partial B\left(p\left(x_{k}\right), r\left(x_{k}\right)\right)\right) \backslash B\left(x_{k}, \varepsilon\right) \subset B\left(p\left(y_{k}\right), r\left(y_{k}\right)\right) \quad \text { for } k \geq k_{0} .
$$

Proof. Let $\varepsilon>0$ and put

$$
\Gamma_{k} \equiv \partial B\left(p\left(x_{k}\right), r\left(x_{k}\right)\right) \backslash B\left(x_{k}, \varepsilon\right) .
$$

If $\Gamma_{k}=\emptyset$, then the lemma holds trivially. Assume that $\Gamma_{k} \neq \emptyset$. For $x \in \Gamma_{k}$, we denote the angle between $x-p\left(x_{k}\right)$ and $n\left(x_{k}\right)$ by $\alpha_{k}(x)$ and the angle between $x-p\left(x_{k}\right)$ and $p\left(y_{k}\right)-p\left(x_{k}\right)$ by $\beta_{k}(x)$. Then there is a $\delta>0$ by the definition of $\Gamma_{k}$ such that

$$
\left(\frac{x-p\left(x_{k}\right)}{\left|x-p\left(x_{k}\right)\right|}, n\left(x_{k}\right)\right)=\cos \alpha_{k}(x) \geq-1+2 \delta,
$$

for $x \in \Gamma_{k}$. Here $\delta$ is independent of $k$ and $x$. Using Lemma 7.7 and (7.27), we estimate $\cos \beta_{k}(x)$ as

$$
\begin{aligned}
\cos \beta_{k}(x)= & \left(\frac{x-p\left(x_{k}\right)}{\left|x-p\left(x_{k}\right)\right|}, \frac{p\left(y_{k}\right)-p\left(x_{k}\right)}{\left|p\left(y_{k}\right)-p\left(x_{k}\right)\right|}\right) \\
= & \left(\frac{x-p\left(x_{k}\right)}{\left|x-p\left(x_{k}\right)\right|}, n\left(x_{k}\right)\right) \\
& \quad+\left(\frac{x-p\left(x_{k}\right)}{\left|x-p\left(x_{k}\right)\right|}, \frac{p\left(y_{k}\right)-p\left(x_{k}\right)}{\left|p\left(y_{k}\right)-p\left(x_{k}\right)\right|}-n\left(x_{k}\right)\right) \\
\geq & -1+\delta,
\end{aligned}
$$

for $k$ large enough. By (7.24), there is a positive sequence $\left\{\delta_{k}\right\}$ converging to zero such that

$$
\left|p\left(y_{k}\right)-p\left(x_{k}\right)\right| \leq\left(1+\delta_{k}\right)\left(r\left(y_{k}\right)-r\left(x_{k}\right)\right) .
$$

Since $\left\{x_{k}\right\}$ and $\left\{y_{k}\right\}$ have the common limit $x_{0}$, the ratio $r\left(x_{k}\right) / r\left(y_{k}\right)$ converges to 1. Then we choose $k_{0} \in \mathbb{N}$ so large that both (7.28) and the conditions below hold for $k \geq k_{0}$ :

$$
\begin{gathered}
p\left(x_{k}\right) \in B\left(p\left(y_{k}\right), r\left(y_{k}\right)\right), \\
\frac{1}{2}<\frac{r\left(x_{k}\right)}{r\left(y_{k}\right)}<1, \\
\delta+\delta \delta_{k}-2 \delta_{k}-\delta_{k}^{2} / 2>0 .
\end{gathered}
$$


We show (7.26). Let $k \geq k_{0}$ and $x \in \Gamma_{k}$. We apply the law of cosines to the triangle with vertices $x, p\left(x_{k}\right)$ and $p\left(y_{k}\right)$ and use (7.28), (7.29). Then we have

$$
\begin{aligned}
& \left|p\left(y_{k}\right)-x\right|^{2}=\left|x-p\left(x_{k}\right)\right|^{2}+\left|p\left(y_{k}\right)-p\left(x_{k}\right)\right|^{2} \\
& -2\left|x-p\left(x_{k}\right)\right|\left|p\left(y_{k}\right)-p\left(x_{k}\right)\right| \cos \beta_{k}(x) \\
& \leq r\left(x_{k}\right)^{2}+\left(1+\delta_{k}\right)^{2}\left(r\left(y_{k}\right)-r\left(x_{k}\right)\right)^{2} \\
& +2(1-\delta)\left(1+\delta_{k}\right) r\left(x_{k}\right)\left(r\left(y_{k}\right)-r\left(x_{k}\right)\right) \\
& =\left(2 \delta+2 \delta \delta_{k}+\delta_{k}^{2}\right) r\left(x_{k}\right)^{2}-2\left(1+\delta_{k}\right)\left(\delta+\delta_{k}\right) r\left(x_{k}\right) r\left(y_{k}\right) \\
& +\left(1+2 \delta_{k}+\delta_{k}^{2}\right) r\left(y_{k}\right)^{2} .
\end{aligned}
$$

We show that the right-hand side is less than $r\left(y_{k}\right)^{2}$. Subtract $r\left(y_{k}\right)^{2}$ from the right-hand side, divide it by $r\left(y_{k}\right)^{2}$ and put $t=r\left(x_{k}\right) / r\left(y_{k}\right)$ and define

$$
g(t) \equiv\left(2 \delta+2 \delta \delta_{k}+\delta_{k}^{2}\right) t^{2}-2\left(1+\delta_{k}\right)\left(\delta+\delta_{k}\right) t+2 \delta_{k}+\delta_{k}^{2} .
$$

Note that $t=r\left(x_{k}\right) / r\left(y_{k}\right) \in(1 / 2,1)$ by $(7.31)$. To get the conclusion, it is enough to show that $g(t)<0$ for $t \in(1 / 2,1)$. From easy calculations with $(7.32)$, it follows that $g(1)=0$ and

$$
\begin{gathered}
g^{\prime}(1)=2\left(\delta+\delta \delta_{k}-\delta_{k}\right)>0, \\
g(1 / 2)=-\delta / 2-\delta \delta_{k} / 2+\delta_{k}+\delta_{k}^{2} / 4<0 .
\end{gathered}
$$

Then $g(t)<0$ in $(1 / 2,1)$, and the proof is complete.

We are now in a position to prove Lemma 7.5.

Proof of Lemma 7.5. Taking the inner product of (7.15) and $n(x)$, we have

$$
r(x)=(p(x)-x, n(x)) .
$$

Therefore if $p(x)$ is Lipschitz continuous, so is $r(x)$. Let $a>0$. Suppose on the contrary that $p(x)$ is not Lipschitz continuous on $S_{a}$. Then Assumption 7.6 holds. Put $\alpha=r\left(x_{0}\right)+a / 2$. Then there is a $k_{1} \in \mathbb{N}$ such that $r\left(x_{k}\right)<\alpha$ for $k \geq k_{1}$. Since $x_{0} \in S_{a}, \alpha$ is less than $R\left(x_{0}\right)$, then there is a $\delta>0$ by Lemma 7.3 (i) such that

$$
B(x+\alpha n(x), \alpha) \cap B(x, \delta) \subset D,
$$

for $x \in B\left(x_{0}, \delta\right) \cap \partial D$. Hence we can choose $k_{2}\left(\geq k_{1}\right)$ so large that

$$
B\left(x_{k}+\alpha n\left(x_{k}\right), \alpha\right) \cap B\left(x_{k}, \delta\right) \subset D,
$$

for $k \geq k_{2}$. For $\delta$ above, we choose $k_{3}\left(\geq k_{2}\right)$ by Lemma 7.8 such that

$$
\Gamma_{k} \equiv \partial B\left(p\left(x_{k}\right), r\left(x_{k}\right)\right) \backslash B\left(x_{k}, \delta\right) \subset B\left(p\left(y_{k}\right), r\left(y_{k}\right)\right),
$$

for $k \geq k_{3}$. We fix $k$ as $k=k_{3}$. Since $\Gamma_{k}$ is compact and $B\left(p\left(y_{k}\right), r\left(y_{k}\right)\right)$ is open, there is a $\beta \in\left(r\left(x_{k}\right), \alpha\right)$ slightly larger than $r\left(x_{k}\right)$ such that

$$
\partial B\left(x_{k}+\beta n\left(x_{k}\right), \beta\right) \backslash B\left(x_{k}, \delta\right) \subset B\left(p\left(y_{k}\right), r\left(y_{k}\right)\right) \subset D .
$$

Observe that the convex hull of

$$
\left(B\left(x_{k}+\alpha n\left(x_{k}\right), \alpha\right) \cap B\left(x_{k}, \delta\right)\right) \cup\left(\partial B\left(x_{k}+\beta n\left(x_{k}\right), \beta\right) \backslash B\left(x_{k}, \delta\right)\right)
$$

includes $B\left(x_{k}+\beta n\left(x_{k}\right), \beta\right)$. Since $D$ is convex, we obtain

$$
B\left(x_{k}+\beta n\left(x_{k}\right), \beta\right) \subset D .
$$

However, this is impossible because $r\left(x_{k}\right)<\beta$. Consequently, $p(x)$ is Lipschitz continuous on $S_{a}$ and so is $r(x)$. 
Recall that we denote the $\mathbb{R}^{N}$-Lebesgue measure by $\operatorname{vol}(\cdot)$.

Lemma 7.9. $\operatorname{vol}(p(\partial D))=0$.

Proof. For $k \in \mathbb{N}$, we put

$$
\begin{gathered}
\partial D_{k} \equiv\{x \in \partial D: r(x) \leq R(x)-1 / k\}, \\
\partial D_{0} \equiv\{x \in \partial D: r(x)=R(x)\} .
\end{gathered}
$$

Then $\partial D=\bigcup_{k=0}^{\infty} \partial D_{k}$. By Lemma 7.5, $p(x)$ is Lipschitz continuous on $\partial D_{k}$ for $k \geq 1$. Since $R(x)=r(x) \leq d$ on $\partial D_{0}$, by Lemma 7.2, $r(x)$ is Lipschitz continuous on $\partial D_{0}$ and so is $p(x)$. Since $\operatorname{vol}\left(\partial D_{k}\right)=0$ and $p(x)$ is Lipschitz continuous, it follows that $\operatorname{vol}\left(p\left(\partial D_{k}\right)\right)=0$. Therefore $p(\partial D)=\bigcup_{k=0}^{\infty} p\left(\partial D_{k}\right)$ has Lebesgue measure zero.

To prove that $\rho(x)$ is smooth in $D$ except for $p(\partial D)$, we prove the lemma below.

Lemma 7.10. Let $x_{0} \in \partial D, 0<r_{1}<r\left(x_{0}\right)$ and put $x_{1}=x_{0}+r_{1} n\left(x_{0}\right)$. Then $\rho(x)$ belongs to $C^{\infty}$ in a neighborhood of $x_{1}$ and $|\nabla \rho(x)|=1$.

Proof. Let $U$ be an open neighborhood of $x_{0}$ such that $\partial D \backslash U \neq \emptyset$. Put $\Gamma \equiv \partial D \cap U$. We show that there exists a $\delta>0$ such that

$$
\operatorname{dist}(x, \partial D)=\operatorname{dist}(x, \Gamma) \text { for } x \in B\left(x_{1}, \delta\right) \text {. }
$$

Let $y \in \partial D \backslash \Gamma$ and $L$ be the line segment connecting $x_{1}$ with $y$. Denote $L \cap$ $\partial B\left(x_{1}, r_{1}\right)$ and $L \cap \partial B\left(p\left(x_{0}\right), r\left(x_{0}\right)\right)$ by $z_{1}(y)$ and $z_{2}(y)$, respectively. Since these two spheres are tangent at $x_{0}$ only, we have

$$
\min _{y \in \overline{\partial D \backslash \Gamma}}\left|z_{1}(y)-z_{2}(y)\right|\left(\equiv \rho_{0}\right)>0
$$

We show (7.35). For $x \in B\left(x_{1}, \delta\right)$ and $y \in \partial D \backslash \Gamma$, we have

$$
\begin{aligned}
|x-y| & \geq\left|x_{1}-y\right|-\left|x-x_{1}\right| \\
& \geq\left|x_{1}-z_{1}\right|+\left|z_{1}-z_{2}\right|-\left|x-x_{1}\right| \\
& \geq r_{1}+\rho_{0}-\delta .
\end{aligned}
$$

This gives

$$
\operatorname{dist}(x, \partial D \backslash \Gamma) \geq r_{1}+\rho_{0}-\delta, \quad \text { for } x \in B\left(x_{1}, \delta\right) .
$$

On the other hand, we get

$$
\operatorname{dist}(x, \Gamma) \leq\left|x-x_{1}\right|+\operatorname{dist}\left(x_{1}, \Gamma\right) \leq \delta+r_{1}, \quad \text { for } x \in B\left(x_{1}, \delta\right) .
$$

Therefore, for $\delta>0$ small enough, we have

$$
\operatorname{dist}(x, \partial D \backslash \Gamma)>\operatorname{dist}(x, \Gamma), \quad \text { for } x \in B\left(x_{1}, \delta\right) .
$$

This means (7.35).

By translating and rotating the coordinate system, we can assume that $x_{0}=0$ and (7.1)-(7.3) hold. Put $\Gamma \equiv \partial D \cap U$. Then we choose $\delta>0$ which satisfies (7.35). Define $u\left(x^{\prime}\right)$ and $n\left(x^{\prime}\right)$ by (7.4) and (7.6), respectively. Note that $r\left(x_{0}\right)=r(0)$ because we assume $x_{0}=0$. For $t \in(0, r(0))$ and $x^{\prime} \in B_{N-1}(0, \varepsilon)$, we define

$$
\psi\left(x^{\prime}, t\right) \equiv u\left(x^{\prime}\right)+t n\left(x^{\prime}\right)
$$


We show that the Jacobian of $\psi$ is positive. Denote the $i$-th element of $\psi$ by $\psi_{i}$. Then

$$
\begin{gathered}
\psi_{i}\left(x^{\prime}, t\right)=x_{i}-t \phi_{x_{i}}\left(x^{\prime}\right)\left(|\nabla \phi|^{2}+1\right)^{-1 / 2}, \quad \text { for } 1 \leq i \leq N-1, \\
\psi_{N}\left(x^{\prime}, t\right)=\phi\left(x^{\prime}\right)+t\left(|\nabla \phi|^{2}+1\right)^{-1 / 2} .
\end{gathered}
$$

Since $\phi(0, \ldots, 0)=0$ and $\nabla \phi(0, \ldots, 0)=(0, \ldots, 0)$, we have

$$
\begin{gathered}
\frac{\partial \psi_{i}}{\partial x_{j}}(0, t)=\delta_{i j}-t \phi_{x_{i} x_{j}}(0), \\
\frac{\partial \psi_{N}}{\partial x_{j}}(0, t)=0, \quad \frac{\partial \psi_{i}}{\partial t}(0, t)=0, \quad \frac{\partial \psi_{N}}{\partial t}(0, t)=1 .
\end{gathered}
$$

Hence the Jacobian is

$$
\frac{\partial \psi}{\partial\left(x^{\prime}, t\right)}(0, t)=\operatorname{det}(I-t H(0))
$$

Here $I$ stands for the unit matrix and $H(0)$ denotes the Hessian matrix,

$$
H(0)=\left(\phi_{x_{i} x_{j}}(0)\right)_{i j} .
$$

Recall that $G\left(x^{\prime}\right)$ is defined by (7.5). Since $G(0)$ is the unit matrix, the principal curvatures coincide with the eigenvalues of $H(0)$. Denote them by

$$
(0 \leq) \lambda_{1}(0) \leq \cdots \leq \lambda_{N-1}(0) .
$$

Then we compute (7.36) as

$$
\operatorname{det}(I-t H(0))=\left(1-t \lambda_{1}(0)\right) \cdots\left(1-t \lambda_{N-1}(0)\right) .
$$

For $t \in(0, r(0))$, it holds that $t<r(0) \leq R(0)$ and hence $1 / t>\Lambda(0)=\max _{i} \lambda_{i}(0)$. Therefore (7.37) is positive. Since $\psi$ is of class $C^{\infty}$ near $(0, t)$, the Jacobian is positive near $(0, t)$. Put $y=\psi\left(x^{\prime}, t\right)$. By the inverse function theorem, $\left(x^{\prime}, t\right)$ is a $C^{\infty}$ function of $y$, in particular $t=t(y)$ is of class $C^{\infty}$. Consequently,

$$
t(y)=\operatorname{dist}(y, \Gamma)=\operatorname{dist}(y, \partial D)
$$

belongs to $C^{\infty}$. We can prove $|\nabla \rho(x)|=1$ in the same way as in the proof of Lemma 5.1. The proof is complete.

We conclude this paper by proving Theorem 6.3 .

Proof of Theorem 6.3. Since $p(x)$ is continuous on $\partial D, K=p(\partial D)$ is compact. By Lemma $7.9, \operatorname{vol}(p(\partial D))=0$. It remains only to show that $\rho \in C^{\infty}(D \backslash K)$ and $|\nabla \rho(x)|=1$. Let $x_{1} \in D \backslash K$ and $B\left(x_{1}, r_{1}\right)$ be the maximum ball centered at $x_{1}$ that is contained in $D$. Choose a point $x_{0}$ such that $x_{0} \in \partial B\left(x_{1}, r_{1}\right) \cap \partial D$. Then $x_{1}$ is represented as $x_{1}=x_{0}+r_{1} n\left(x_{0}\right)$. Then $r_{1}$ is less than $r\left(x_{0}\right)$ because $x_{1} \notin K$. By Lemma 7.10, $\rho(x)$ belongs to $C^{\infty}$ in a neighborhood of $x_{1}$ and $|\nabla \rho(x)|=1$.

\section{REFERENCES}

1. H. Amann, Fixed point equations and nonlinear eigenvalue problems in ordered Banach spaces, SIAM Rev. 18 (1976) 620-709. MR0415432(54:3519)

2. J. Bao, Positive solution for semilinear elliptic equation on general domain, Nonlinear Anal. 53 (2003) 1179-1191. MR.1978042 (2004a:35058)

3. H. Brezis and L. Oswald, Remarks on sublinear elliptic equations, Nonlinear Anal. 10 (1986) 55-64. MR 820658 (87c:35057)

4. D.G. de Figueiredo, Positive solutions of semilinear elliptic problems, Lecture Notes in Mathematics, Vol. 957, Springer, Berlin, 1982, 34-87. MR679140 (84k:35067) 
5. D. Gilbarg and N. S. Trudinger, Elliptic partial differential equations of second order, Springer, Berlin, 1983. MR737190 (86c:35035)

6. R. Kajikiya, Comparison theorem and uniqueness of positive solutions for sublinear elliptic equations, to appear in Arch. Math.

7. J. W. Milnor, Topology from the differentiable viewpoint, revised edition, Princeton University Press, Princeton, 1997. MR,1487640(98h:57051)

8. K. Taira and K. Umezu, Positive solutions of sublinear elliptic boundary value problems, Nonlinear Anal. 29 (1997) 761-771. MR1455064 (98h:35082)

Nagasaki Institute of Applied Science, 536 Aba-machi, Nagasaki 851-0193, Japan

E-mail address: kajikiya_ryuji@nias.ac.jp

Current address: Department of Mathematics, Faculty of Science and Engineering, Saga University, Saga, 840-8502, Japan

E-mail address: kajikiya@ms.saga-u.ac.jp 